\title{
EquiLíbrio de SolubiLIDADE. UMA DEMONSTRAÇÃO SIMPLES, ADEQUADA AO ENSINO SECUNDÁRIO
}

\author{
Mário Valente*, Helena Moreira*
}

\begin{abstract}
Neste ção de um precipitado de iodeto de chumbo, um sal pouco solúvel, que é convertido em sulfato de chumbo, um sal ainda menos solúvel, por adição de ácido sulfúrico. O precipitado de sulfato de chumbo pode ainda ser reconvertido no iodeto de chumbo por adição de uma solução concentrada de iodeto de potássio. A exploração da experiência sob o ponto de vista teórico é particularmente interessante já que $\mathrm{K}_{\mathrm{s}}\left(\mathrm{PbSO}_{4}\right)>\mathrm{K}_{\mathrm{s}}\left(\mathrm{Pbl}_{2}\right)$ mas $\mathrm{S}\left(\mathrm{PbSO}_{4}\right)<\mathrm{S}\left(\mathrm{Pbl}_{2}\right)$. Este trabalho prático pode ser efectuado com meios rudimentares, em qualquer laboratório escolar.
\end{abstract}

\section{INTRODUÇÃO}

O ensino da química ao nível dos três anos lectivos que constituem o Ensino Secundário tem estado praticamente reduzido a cerca de sete ou oito meses lectivos, contidos na disciplina bianual de Física e Química A.

Esta situação foi agravada pelo facto de a disciplina de Química $\left(12^{\circ}\right.$ ano) ter sido despromovida a disciplina de carácter opcional, o que teve como consequência o seu desaparecimento quase completo a nível nacional. A partir deste ano lectivo de 2009/2010 o novo curriculo do $12^{\circ}$ ano inclui mais uma disciplina de carácter opcional e é de esperar que o ensino da química recupere um pouco do que têm sido anos complicados.

O equilíbrio de solubilidade é um dos conceitos integrantes do programa da disciplina de Física e Química A, nível 2 [1], nos quais os alunos mostram maiores dificuldades. De acordo com a nossa prática lectiva, estas dificuldades são frequentemente diminuidas pela realização, por parte dos alunos, de actividades práticas, em paralelo com a exposição teórica da matéria.

De seguida descreve-se a realização de uma actividade laboratorial simples mas que permite esclarecer/consolidar algumas características importantes do equilíbrio de solubilidade.

* Colégio D. Duarte, Rua Visconde de Setúbal, 86, Porto, madmage1@yahoo.com

\section{Prática de Laboratório}

A experiência proposta neste artigo faz uso de soluções aquosas de nitrato de chumbo e de iodeto de potássio, ambas a $1 \%(\mathrm{~m} / \mathrm{m})$, de uma solução aquosa de iodeto de potássio a $20 \%$ $(\mathrm{m} / \mathrm{m})$ e de uma solução aquosa de ácido sulfúrico a $30 \%(\mathrm{~m} / \mathrm{m})-$ ver Nota.

\section{EXPERIÊNCIA 1:}

\section{PrecipitaÇÃo do IODETO dE CHUMBO}

A cerca de três mililitros de solução de nitrato de chumbo a $1 \%$ adiciona-se igual volume de solução de iodeto de potássio a $1 \%$, com o que se verifica a formação de um precipitado amarelo característico do iodeto de chumbo, um sal pouco solúvel em água (Figura $1 \mathrm{~A}$ ). O processo pode ser traduzido pela seguinte equação:

$\mathrm{Pb}^{2+}(\mathrm{aq})+2 \mathrm{I}^{-}(\mathrm{aq}) \leftrightarrows \mathrm{Pbl}_{2}(\mathrm{~s})$

[eq.1]

A precipitação verifica-se por que, após a mistura, a solução resultante contém os iões $\mathrm{Pb}^{2+}, \mathrm{K}^{+}, \mathrm{I}^{-}$e $\mathrm{NO}_{3}^{-}$. Ao contrário de outras combinações possíveis $\left(\mathrm{Pb}^{2+} / \mathrm{NO}_{3}^{-}, \mathrm{K}^{+} / \mathrm{NO}_{3}^{-}\right.$e $\left.\mathrm{K}^{+} / \mathrm{I}^{-}\right)$ os iões $\mathrm{Pb}^{2+} \mathrm{e} \mathrm{I}^{-}$formam entre si um composto pouco solúvel, o iodeto de chumbo $\mathrm{Pbl}_{2}$.

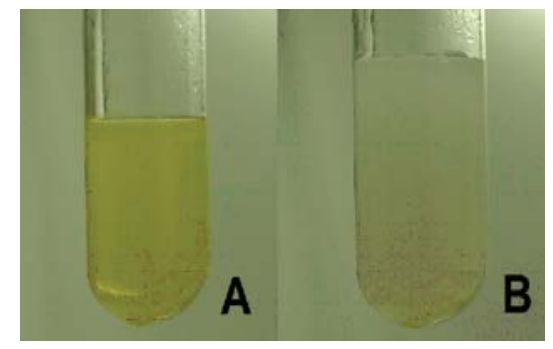

Figura 1 Tubos de ensaio contendo suspensões dos precipitados (A) de iodeto de chumbo e (B) de sulfato de chumbo em água
A expressão da constante de equilíbrio para o processo de solubilização (inverso do acima apresentado) pode ser escrita segundo:

$\mathrm{K}_{\mathrm{s}}\left(\mathrm{Pbl}_{2}\right)=\left[\mathrm{Pb}^{2+}\right]_{\mathrm{e}}\left[\mathrm{I}^{-}\right]_{\mathrm{e}}^{2}$

[exp.1]

e tem o valor de $7,9 \times 10^{-9}$ a $25^{\circ} \mathrm{C}$ [2]. Da expressão 1 deduz-se facilmente que a solubilidade do iodeto de chumbo é dada pela expressão: 
A expressão da respectiva constante de equilíbrio vem escrita pela expressão:

$$
\mathrm{K}_{\mathrm{s}}\left(\mathrm{PbSO}_{4}\right)=\left[\mathrm{Pb}^{2+}\right]_{\mathrm{e}}\left[\mathrm{SO}_{4}^{2-}\right]_{\mathrm{e}} \quad[\exp .3]
$$

e tem o valor de $1,6 \times 10^{-8}$ a $25^{\circ} \mathrm{C}$ [2], de que resulta uma solubilidade dada pela expressão:

$$
\mathrm{S}\left(\mathrm{PbSO}_{4}\right)=\sqrt{\mathrm{K}_{\mathrm{s}}\left(\mathrm{PbSO}_{4}\right)} \quad \text { [exp.4] }
$$

já que $\left[\mathrm{Pb}^{2+}\right]_{e}=\mathrm{S}\left(\mathrm{PbSO}_{4}\right)$ e que $\left[\mathrm{SO}_{4}{ }^{2-}\right]_{\mathrm{e}}=\mathrm{S}\left(\mathrm{PbSO}_{4}\right)$. Substituíndo $\mathrm{K}_{\mathrm{s}}\left(\mathrm{PbSO}_{4}\right)$ pelo seu valor chega-se a uma solubilidade, $\mathrm{S}\left(\mathrm{PbSO}_{4}\right)$, de $1,3 \times 10^{-4} \mathrm{~mol} / \mathrm{dm}^{3}$. Importa salientar aos alunos que a solubilidade do sulfato de chumbo é inferior à do iodeto de chumbo apesar dos valores dos correspondentes produtos de solubilidade apresentarem uma ordem inversa, o que lhes permite concluir que, dependendo da estequiometria entre os aniões e os catiões que constituem o sal, nem sempre a ordenação das solubilidades pode ser directamente feita olhando aos valores dos produtos de solubilidade.

\section{EXPERIÊNCIA 2:}

\section{Conversão dO IODEtO de Chumbo em SULFATO de ChumbO}

Decanta-se o líquido sobrenadante resultante da experiência 1 para um frasco de restos de sais de chumbo. Adiciona-se ao precipitado um volume de cerca de seis mililitros de água desionizada e agita-se de modo a suspender o precipitado. Passa-se metade da suspensão resultante para outro tubo de ensaio e adicionam-se-lhe cerca de três mililitros de solução de ácido sulfúrico a 33\%. Verifica-se uma alteração na cor do precipitado que passa de amarelo para branco (Figura $1 \mathrm{~B}$ ), devido à conversão do iodeto de chumbo em sulfato de chumbo:

$\mathrm{Pbl}_{2}(\mathrm{~s})+\mathrm{H}_{2} \mathrm{SO}_{4}(\mathrm{aq}) \leftrightarrows \mathrm{PbSO}_{4}(\mathrm{~s})+2 \mathrm{HI}(\mathrm{aq})$

[eq.3]

Nota: Pode ser necessário aquecer a suspensão para acelerar o processo.

O processo que ocorre na experiência 2 poderia ser explicado meramente com base na solubilidade inferior do sulfato de chumbo relativamente ao iodeto de chumbo. Tomando em consideração o princípio de Le Chatelier é, contudo, possível reconverter o precipitado de sulfato de chumbo (branco) em precipitado de iodeto de chumbo (amarelo) por adição de uma solução concentrada de iodeto de potássio - é o que se sugere na experiência 3 .

Apesar de o sulfato de chumbo ser, dos dois, o sal menos solúvel, por adição de um grande excesso de anião iodeto favorece-se a formação de iodeto de chumbo e a cor do precipitado volta a ser amarela. compreensão da teoria subjacente, em particular quando são acompanhados de uma exploração pertinente do que se observa. Esta realidade não pode ser mais verdadeira do que no caso das ciências naturais em geral e da química em particular, especialmente no caso do presente currículo do ensino secundário, no qual a prática aparece como acessória da teoria (e até, por vezes, completamente desligada).

Consideramos o trabalho proposto como útil, já que proporciona uma ligação eficaz entre conceitos de equilíbrio químico como o princípio de Le Chatelier e a prática de laboratório.
Nota: A preparação destas soluções deve ser realizada pelo professor. As soluções de nitrato de chumbo e de iodeto de potássio a $1 \%$ podem ser preparadas dissolvendo $1,0 \mathrm{~g}$ de cada sal em água desionizada, até um volume total de $100 \mathrm{ml}$. A preparação da solução de iodeto de potássio a $20 \%$ pode ser facilmente conseguida dissolvendo 2,0 g deste sal em $8 \mathrm{ml}$ de água. A preparação da solução de ácido sulfúrico requer cuidados acrescidos, quer por que este é extremamente corrosivo, quer por que a sua solubilização é fortemente exotérmica. Assim, para se preparar a referida solução deve adicionar-se lentamente e com agitação constante, um volume de cerca de $20 \mathrm{ml}$ de ácido sulfúrico concentrado, a um volume de cerca de $60 \mathrm{ml}$ de água desionizada contido num gobelé de $250 \mathrm{ml}$. Durante a preparação desta solução é de grande conveniência ter à mão papel absorvente para limpar quaisquer gotas de ácido sulfúrico concentrado que caiam na mesa, que escorram da sua garrafa ou (sucedendo o pior) que caiam na pele - neste último caso só depois de limpar com o papel absorvente é que se pode lavar a zona afectada com sabão e água.

Nota de segurança: De uma forma geral os sais de chumbo são tóxicos, em particular os mais solúveis, como o nitrato. Assim, ao lidar com soluções de sais de chumbo (mesmo as diluídas) devem usar-se luvas. A solução de ácido sulfúrico a $33 \%$ é ainda bastante corrosiva, pelo que durante a sua utilização se devem usar luvas e óculos de protecção.

\section{Bibliografia}

1) Programa da disciplina de Física e Química A (nível 2) $11^{\circ}$ ou $12^{\circ}$ anos: http:// www.dgidc.min-edu.pt/programs/prog_ eg.asp

2) João Cabral, Tabelas e Formulário de Química, Universidade do Porto, Porto 1993. 


\section{Actualidades Científicas}

\section{Quanto Mais Pequeno, Melhor!}

De acordo com uma equipa de investigação japonesa, é possível sintetizar partículas metálicas de $1 \mathrm{~nm}$ e mesmo subnanométricas, contendo um número bem definido de átomos, através de um método baseado no conceito de dendrímero. A equipa descreve um processo inovador de síntese de partículas com diâmetro na gama que ronda $1 \mathrm{~nm}$ e reporta que estas apresentam uma actividade catalítica superior ao que seria expectável tendo em consideração conclusões anteriores. (Nat. Chem., DOI: 10.1038/ nchem.288).

Esta questão é importante já que, por exemplo, em algumas aplicações específicas, se sabe que partículas de 3 $\mathrm{nm}$ de diâmetro são mais activas cataliticamente do que as suas correspondentes com o dobro do tamanho e, deste modo, se poderia especular que partículas de tamanho mais baixo (1 ou $0,5 \mathrm{~nm}$ ) ainda deveriam ser melhores do ponto de vista catalítico. Contudo, as evidências experimentais até agora reunidas contrariam esta conclusão. Por exemplo, para a reacção de redução de oxigénio (oxygen-reduction reaction - ORR), o tamanho óptimo para as partículas de catalisador de platina corresponderia a aproximadamente $3 \mathrm{~nm}$. No entanto, Kimihisa Yamamoto, Takane Imaoka e colaboradores da Universidade de Keio, anunciam agora que a actividade catalítica de catalisadores ORR de platina aumenta com o decréscimo do tamanho das partículas até à gama de tamanhos inferior a $1 \mathrm{~nm}$.

De modo a sintetizar partículas nesta gama de tamanhos, que se tem revelado particularmente difícil de atingir de uma forma controlada, os investigadores desenvolveram um novo dendrímero baseado em unidades de fenilazometino, que forma complexos numa relação de um para um com sais de platina. Assim, através do tratamento sequenciado de várias amostras de solução de dendrímero com uma quantidade seleccionada equivalente de cloreto de platina, a equipa conseguiu produzir dendrímeros contendo um número crescente de átomos de platina. Posteriormente, quando o produto obtido é reduzido, os átomos de platina coalescem em núcleos de tamanho pré-definido, no interior dos dendrímeros.
Através da caracterização por análise microscópica, a equipa verificou que o procedimento conduziu à síntese de núcleos (ou partículas) com diâmetros de 0,$9 ; 1,0$ e $1,2 \mathrm{~nm}$, constituídos por 12,28 e 60 átomos, respectivamente. Testes catalíticos de ORR mostram que o núcleo mais activo é o mais pequeno e que o catalisador de $0,9 \mathrm{~nm}$ é 13 vezes mais activo do que um catalisador comercial de platina de 2,5 nm, o que, segundo Yamamoto, "contraria o pensamento actual dominante".

Richard M. Crooks, professor de Química da Universidade do Texas, Austin, EUA, acrescenta "Apesar de ser claro que os procedimentos baseados em dendrímeros são bastante promissores do ponto de vista da síntese, ainda resta muito trabalho a realizar no sentido da compreensão da relação entre o tamanho das nanopartículas e a sua actividade electrocatalítica. Este trabalho representa um passo estimulante na prossecução desse objectivo".

(adaptado de Chemical \& Engineering News 87 (30) (2009) 12)

Paulo Brito
Novo REAGENTE PARA DETECÇÃo E DECOMPOSIÇÃO DE GASES NEUROTÓXICOS

Os gases neurotóxicos (gases de nervos), como Soman, Tabun e Sarin (utilizado no ataque terrorista no Metro de Tóquio em 1995) podem ser letais em alguns minutos se forem inalados. Por isso, têm sido investigados vários métodos e sistemas de detecção que, contudo, apresentam limitações, como baixa sensibilidade, complexidade operacional e não podem ser usados in loco. Num trabalho publicado recentemente na Angewandte Chemie International Edition (DOI: 10.1002/ anie.200902820), dois investigadores do The Scripps Research Institute em La Jolla, Califórnia (Julius Rebek, Jr. e Trevor Dale), descrevem uma nova classe de sensores, que respondem muito mais rapidamente que os anteriormente conhecidos, exibem maior sensibilidade e, além disso, promo- vem a sua degradação. Os gases de nervos são todos organofosfatos:
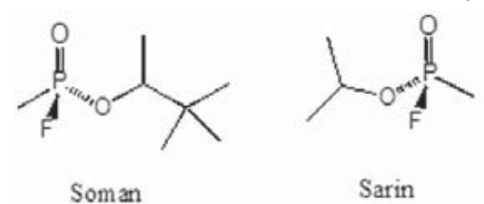

dá uma ciclização, com libertação de um produto não tóxico, como se ilus-

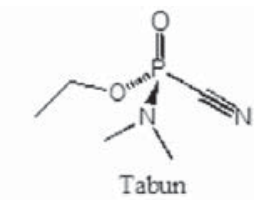

Os compostos preparados por Rebek e Dale consistem em sistemas aromáticos com um grupo oxima $(-\mathrm{C}=\mathrm{N}$ $\mathrm{OH})$ que se liga a organofosfatos de forma extremamente rápida. Contudo, o produto resultante é ele próprio tão tóxico como o gás. A presença de um grupo hidroxilo em posição orto em relação ao grupo oxima permite que ocorra um segundo passo em que se

tra no esquema abaixo. Os compostos de partida ( $\beta$-hidroxioximas) usados são fluorescentes e os autores verificaram ainda que o produto final é também fuorescente, emitindo a um comprimento de onda diferente. Além disso, há um aumento do rendimento de fluorescência. Esta aumenta 62 vezes para o derivado naftalénico e 46 vezes para o derivado do pireno.

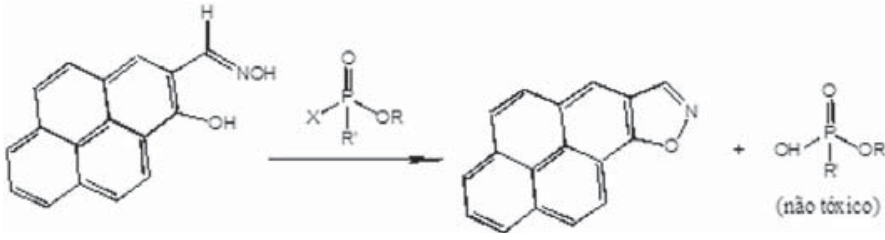
JM 
Most accessed articles

9/2006 - 8/2007

A General and Facile One-Step Synthesis of Imido-Titanium(IV) Complexes: Application to the Synthesis of Compounds Containing Functionalized or Chiral Imido Ligands and Bimetallic Diimido Architectures

C. Lorber, R. Choukroun, L. Vendier

Eur. J. Inorg. Chem. 2006, 4503-4518

Classical and Non-Classical Ruthenium-

Based Anticancer Drugs: Towards Targeted Chemotherapy

W. H. Ang, P. J. Dyson

Eur. J. Inorg. Chem. 2006, 4003-4018

Nanoscopic Metal Particles - Synthetic Methods and Potential Applications

H. Bönnemann, R. M. Richards

Eur. J. Inorg. Chem. 2001, 2455-2480

Nanoparticulated Gold: Syntheses, Structures, Electronics, and Reactivities G. Schmid, B. Corain

Eur. J. Inorg. Chem. 2003, 3081-3098

Osmium- and Ruthenium-Based Phosphorescent Materials: Design, Photophysics, and Utilization in OLED Fabrication P. - T. Chou, Y. Chi

Eur. J. Inorg. Chem. 2006, 3319-3332

Full-text downloads have more than doubled since 2003. Eur/IC is among the top ten most-cited primary journals in inorganic chemistry.

For more information please visit: www.eurjic.org

Subscribe now! Please send an e-mail to: cs-journals@wiley.com

(North and South America)

service@wiley-vch.de

(Germany/Austria/Switzerland)

cs-journals@wiley.co.uk

(all other areas)

\section{WILEY}

\section{InterScience ${ }^{\circ}$}

WWILEY-VCH

\section{Made in Europe for the World}

\section{EurJIC Facts}

- Short Communications median time from submission to web publication 10 weeks

- Author submissions have more than doubled since 2001

- Steady increase in impact since the amalgamation of ten national society journals

- Table of Contents redesigned in 2007 to assist browsing

- Templates available for submission of articles

- Increased frequency in 2007: 36 issues

- Owned and supported by 13 national chemical societies in Europe (Editorial Union of Chemical Societies)

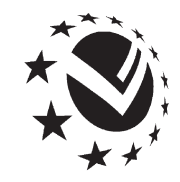

EUChemSoc

\section{EurJIC offers:}

- RSS feeds 1

- Citation tracking

- Table of Contents Alerts

- Backfiles of Chemische Berichte (1868-1997) as part of the Chemical Societies Backfiles Collection

- Attractive personal subscription rates for society members

\section{International interest in EurJIC as reflected by} the percentage of downloads from each country

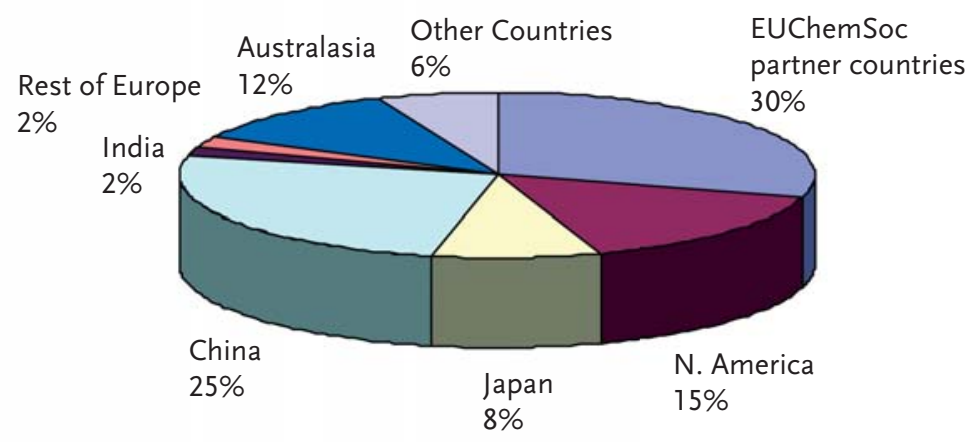

Impact Factors announced in the year given

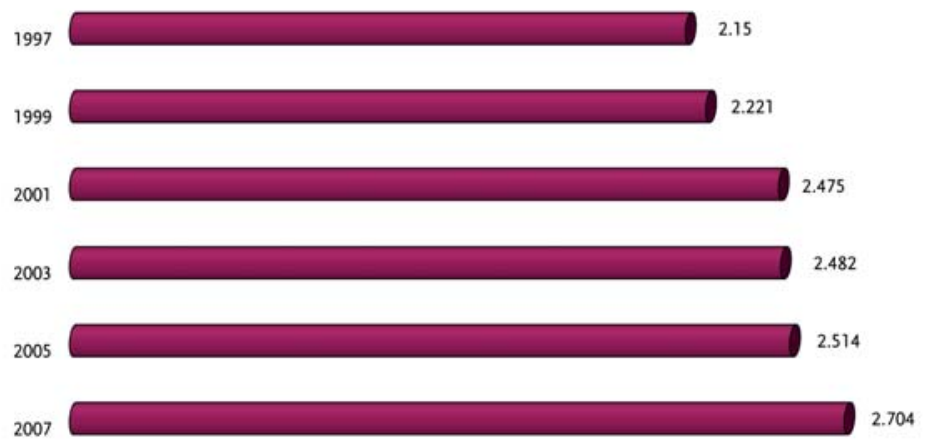

